\title{
Investigation of Salivary RANKL and OPG Levels in Periodontitis Patients at Hospital Universiti Sains Malaysia
}

\author{
Saba Asif ${ }^{1}$ Basaruddin Ahmad ${ }^{1}$ Syed Ameer Hamza ${ }^{2}$ Haslina Taib ${ }^{1} \quad$ Nur Karyatee Kassim ${ }^{1}$ \\ Siti Lailatul Akmar Zainuddin ${ }^{1}$
}

${ }^{1}$ School of Dental Sciences, Health Campus, Universiti Sains

Malaysia, Kelantan, Malaysia

${ }^{2}$ Department of Oral Medicine, Dental Hospital, The University of Faisalabad, Pakistan

\begin{abstract}
Address for correspondence Siti Lailatul Akmar Zainuddin, PhD, School of Dental Sciences, Health Campus, Universiti Sains Malaysia, 16150 Kubang Kerian, Kelantan, Malaysia (e-mail: lailatul@usm.my).
\end{abstract}

Eur J Dent 2022;16:173-178.

\begin{abstract}
Keywords

- periodontitis

- receptor activator of NF-kB ligand

- osteoprotegerin

- Saliva

Objective This study was aimed to determine the levels of salivary receptor activator of NF-KB ligand (RANKL) and osteoprotegerin (OPG) and its association with periodontal status among periodontitis patients.

Materials and Methods A cross-sectional study was designed and performed at the Dental Clinic, Hospital Universiti Sains Malaysia (HUSM). Random sampling was employed to identify 88 participants into three groups: 30 mild periodontitis, 30 moderate to severe periodontitis, and 28 healthy (nonperiodontitis) patients. Periodontal parameters: periodontal pocket depth (PPD), clinical attachment level (CAL), plaque score (PS), and gingival bleeding index (GBI) were recorded. In total, $4 \mathrm{~mL}$ of unstimulated whole saliva was collected to determine the levels of salivary RANKL and OPG proteins by using ELISA technique. Data were analyzed by using SPSS software version 24.0.

Results Mean values for PPD $(5.3 \pm 0.5)$ and CAL $(5.6 \pm 0.5)$ were observed higher for moderate to severe periodontitis as compared with values $(4.4 \pm 0.2)(4.5 \pm 0.2)$ in mild periodontitis patients. The mean salivary RANKL and OPG was $0.23 \pm 0.07 \mathrm{ng} / \mathrm{mL}$ and $1.78 \pm 0.70 \mathrm{ng} / \mathrm{mL}$ respectively in moderate to severe periodontitis. Only salivary RANKL levels were significantly and positively correlated with all the clinical periodontal parameters.

Conclusion The levels of salivary RANKL were higher as opposed to lower OPG levels in periodontitis patients in contrast to healthy (nonperiodontitis) patients. RANKL levels were significantly associated with the periodontal parameters. Therefore, we can conclude that RANKL can potentially aid as an adjunctive diagnostic protein in evaluating periodontal disease.
\end{abstract}

\section{Introduction}

Periodontal infection is a slow progressive condition responsible for both inflammation as well as undermining of tooth supporting framework as a result of diverse polymicrobial infection and immune responses in susceptible individuals. ${ }^{1}$
Presence of pathogenic anaerobic microorganisms, ${ }^{2}$ plaque biofilm, and host immune reactions are considered as main or primary etiological components for this disease. ${ }^{3}$ In this case, the regulation of disease pathogenesis comprises interactions between immune reactions from the host published online September 27, 2021
DOI https://doi.org/ $10.1055 / \mathrm{s}-0041-1731930$ ISSN 1305-7456 (c) 2021. The Author(s).

This is an open access article published by Thieme under the terms of the Creative Commons Attribution License, permitting unrestricted use, distribution, and reproduction so long as the original work is properly cited. (https:// creativecommons. org/licenses/by/4.0/).

Thieme Medical and Scientific Publishers Pvt. Ltd. A-12, 2nd Floor, Sector 2, Noida-201301 UP, India 
along with body response to the bacterial plaque. ${ }^{4}$ Several prominent mediators of inflammation including interleukin (IL)-1 $\beta$, IL-6, prostaglandin E2, along with tumor necrosis factor (TNF)- $\alpha$, are released within the body, and these substances activate lytic enzymes and cause disintegration of the alveolar bone. ${ }^{5-7}$

NF-kB ligand (RANKL) belonged to the renowned family of TNF receptor, and its activation leads to bone resorption and osteoclast precursor cell differentiation. ${ }^{8}$ RANKL is produced through various cells such as osteoblasts or stromal cells, ${ }^{9}$ fibroblasts, ${ }^{10,11}$ and activated B and T cells. ${ }^{12}$ The surface of osteoclast and preosteoclast cells is covered with RANK receptors that are activated by RANKL. ${ }^{13}$ Binding of RANK with RANKL uplifts the differentiation process for osteoclastic progenitor cells as well as enhances the activity of mature osteoclastic cells. ${ }^{14}$

Osteoprotegerin (OPG) also belongs from TNF receptor family with structural resemblance to RANK. ${ }^{15}$ OPGs are commonly produced via human periodontal ligament (PDL) cells, epithelial cells, and gingival fibroblasts ${ }^{16}$ and further regulated through inflammatory cytokines. ${ }^{17}$ OPG functions as antagonist against RANK specific receptor, competitively binding itself onto the surface of RANK molecules. Thus, not only it halts the coupling of RANKL receptors with RANK, but also impedes the differentiation process for both osteoclasts and osteoclastic activity within the cells. ${ }^{18}$

Bone remodeling (both bone formation and resorption) is a process which is controlled by RANKL, RANK and OPG system since they are considered primary key factor for bone metabolism. ${ }^{19}$ RANK, RANKL, and OPG have been studied for their contribution to the pathological and physiological aspects concerning bone resorption; this has also been researched and discussed previously. ${ }^{20,21}$ Considering the specified reasons, we believe that RANK, OPG, and RANKL have a decisive role in regulating the degeneration of periodontal hard and soft supporting tissue.

Current studies exploring the breakdown of alveolar bone leading to periodontal tissue degeneration demonstrate limitations. Clinical studies evaluating RANKL, OPG levels in gingival crevicular fluid (GCF) interpreted that high RANKL, and low OPG protein levels participate in the underlying process of periodontal tissue breakdown. ${ }^{22-24}$ Interaction of RANKL, OPG proteins with bacterial by products, and inflammatory mediators during periodontitis disease triggers and controls the degenerative process of periodontal tissue breakdown. Till today, none of the previous studies have provided any standard level of RANKL and OPG proteins within whole saliva involved in periodontitis; therefore, this is the first study that was conducted in Malaysian population to evaluate the association of RANKL, OPG proteins using clinical periodontal parameters in both periodontitis and healthy (nonperiodontitis) groups. This study also intended to determine the precise concentrations of both RANKL and OPG proteins in whole saliva from healthy patients and patients with periodontitis. Additionally, we also observed the correlation of these proteins with clinical periodontal parameters in both study groups.

\section{Materials and Methods}

A cross-sectional study was performed at the School of Dental Sciences, Hospital Universiti Sains Malaysia (HUSM). A total of 88 patients from outpatient and periodontics clinic were randomly recruited via probability sampling method and were assigned into three groups: healthy (nonperiodontitis), mild periodontitis, and moderate to severe periodontitis. Patients recruited for this study were already diagnosed with periodontitis. The sample size for salivary RANKL; OPG evaluation in periodontitis patients was calculated by using plaque score (PS) calculator. Calculated sample size for RANKL and OPG protein turned out 27 for each of the three groups using a reference study values. ${ }^{25}$ Keeping in account $10 \%$ drop out rate of patients, the sample size was round off to 30. Patients constituting mild periodontitis and moderate to severe periodontitis were diagnosed based on case definition by Eke et al. ${ }^{26}$ Exclusion criteria consisted of pregnant females, patients having less than 20 permanent teeth, suffering from any systemic disease, on medication, and an antibiotic therapy during the last 3 months. Study approval was obtained and granted by the Human Research Ethics Committee of USM. All patients were made aware of the study, and informed written consent was taken from all patients who wanted to participate.

All the patients were instructed to follow an overnight fast followed by saliva sampling in the morning; during whole procedure, no food or drink was allowed (except water). Prior to clinical measurements, whole saliva samples were obtained and collected via spit out method into the polypropylene tubes. This procedure took about a maximum of 5 minutes for each patient, and both time and volume of collected sample were noted accordingly. ${ }^{27,28}$ Samples incorporating any blood particles were discarded and collected again. A domestic refrigerator was used initially to store the samples within 30 minutes of sampling procedure later on samples were freeze at or below $-20^{\circ} \mathrm{C}$ until the day of assay analysis..$^{28}$

All teeth (except that of all existing third molars) were inspected for all six sites (mesiobuccal, midbuccal, distobuccal, mesiolingual,, midlingual, and distolingual) by one examiner. Periodontal health status was measured by using Michigan 0 periodontal probe, and it comprised of plaque index, periodontal probing depth (PPD), gingival bleeding index (GBI), clinical attachment level (CAL). Intra-examiner agreement using intra class correlation coefficient test was used, and the values for correlation coefficients for PPD and CAL came out to be 0.96 and 0.88 , respectively.

A clean microcap tube containing $500 \mathrm{~mL}$ of individual stored saliva sample was initially clarified by carrying out centrifugation at $10,000 \mathrm{~g}$ for approximately 5 minutes; after which the supernatant was relocated into clean microcap tubes; and then an enzyme linked immunosorbent assay (ELISA) was performed. Commercially available salivary RANKL and OPG ELISA kits from Elabscience (USA) were employed to determine the concentrations of both proteins in the saliva samples corresponding to the manufacturers' manual. Resultant values for salivary RANKL and 
OPG analysis were represented as $\mathrm{ng} / \mathrm{mL}$ for concentrations. Standards were incorporated on all rounds. Moreover, all the data along with results are asserted within the given range of the assays.

Statistical evaluation was executed by using ANOVA test to obtain mean concentrations of OPG and RANKL proteins. Pearson's correlation coefficient analysis was applied to explore the relationship between levels of RANKL and OPG in saliva along with the clinical periodontal parameters. A $p$-value lower than 0.05 was considered statistically significant for this study. This research was conducted by using SPSS software for Windows version 24.0, SPSS Inc., Chicago, Illinois, United States.

\section{Results}

Overall 90 patients participated in the study. However, data of two patients were removed from analysis being incomplete, and data of 88 patients were analyzed. - Table 1 provides an insight into the demographic aspects of the participants who were recruited for this study. It was observed that there was an evident and a significant difference for gender between groups. The periodontitis group was composed mostly of female participants and Malay ethnicity.

In - Table 2, higher mean values for PPD and CAL were observed for patients with severe periodontitis in contrast to mild periodontitis patients. A statistically significant difference was evident among the three study groups in terms of gingival inflammation and mean PS. Moreover, in contrast to other groups in this study, more gingival inflammation and higher mean PSs were observed in the severe periodontitis group.

- Table 3 presents the quantitative determination results for the salivary concentrations of salivary OPG and RANKL. A higher RANKL and OPG levels in severe periodontitis group in contrast with healthy (nonperiodontitis) controls was worth noting in this study.

Intergroup differences were analyzed via Dunnett's formula which are shown in -Table 4 . The mean concentration difference for salivary RANKL was observed to be significant between healthy (nonperiodontitis) to mild periodontitis and healthy (nonperiodontitis) to severe periodontitis group, and a nonsignificant finding for mild periodontitis to severe periodontitis groups protein. However, for OPG, the

Table 1 Demographic characteristics of study patients in healthy, mild and moderate to severe periodontitis groups ( $n=88$ )

\begin{tabular}{|l|l|l|l|}
\hline \multirow{2}{*}{ Group } & \multicolumn{2}{|l|}{ Variables } \\
\cline { 2 - 4 } & $\begin{array}{l}\text { Age }(y) \\
\text { (mean } \pm \text { SD) }\end{array}$ & $\begin{array}{l}\text { Gender } \\
\text { female/male, } \boldsymbol{n}(\%)\end{array}$ & $\begin{array}{l}\text { Race } \\
\text { Malay/Non-Malay } \boldsymbol{n}(\%)\end{array}$ \\
\hline Healthy $(n=28)$ & $30.3(10.2)$ & $\begin{array}{l}15(53.5 \%) \\
13(46.5 \%)\end{array}$ & $\begin{array}{l}19(67.8 \%) \\
9(32.2 \%)\end{array}$ \\
\hline Mild periodontitis $(n=30)$ & $39.1(10.2)$ & $\begin{array}{l}16(53.4 \%) \\
14(46.6 \%)\end{array}$ & $\begin{array}{l}21(70 \%) \\
9(30 \%)\end{array}$ \\
\hline $\begin{array}{l}\text { Moderate-to-severe periodontitis } \\
(n=30)\end{array}$ & $46.0(10.3)$ & $\begin{array}{l}21(70 \%) \\
9(30 \%)\end{array}$ & $\begin{array}{l}21(70 \%) \\
9(30 \%)\end{array}$ \\
\hline$p$-Value $^{\text {d }}$ & & $<0.001$ & $<0.001$ \\
\hline
\end{tabular}

Abbreviation: SD, standard deviation.

aPost hoc test was significant for age between healthy and mild periodontitis group.

bPost hoc test was significant for age between healthy and severe periodontitis group.

'Post hoc test was significant for age between mild and severe periodontitis group.

${ }^{d} p<0.05$ is significant.

Table 2 Periodontal clinical parameters in healthy, mild and moderate to severe periodontitis groups $(n=88)$

\begin{tabular}{|c|c|c|c|c|}
\hline \multirow[t]{2}{*}{ Groups } & \multicolumn{3}{|l|}{ Variables } & \multirow[b]{2}{*}{$\begin{array}{l}\text { Mean clinical } \\
\text { attachment level (mm) }\end{array}$} \\
\hline & $\begin{array}{l}\text { Mean bleeding on } \\
\text { probing (\%) }\end{array}$ & $\begin{array}{l}\text { Mean plaque } \\
\text { scores (\%) }\end{array}$ & $\begin{array}{l}\text { Mean probing } \\
\text { pocket depth }(\mathrm{mm})\end{array}$ & \\
\hline $\begin{array}{l}\text { Healthy (28) } \\
\text { Mean (SD) }\end{array}$ & $11.86 \pm 5.42$ & $20.86 \pm 5.97$ & 00 & 00 \\
\hline $\begin{array}{l}\text { Mild periodontitis (30) } \\
\text { Mean (SD) }\end{array}$ & $51.39 \pm 17.07$ & $46.34 \pm 17.57$ & $4.4 \pm 0.2$ & $4.5 \pm 0.2$ \\
\hline $\begin{array}{l}\text { Moderate-to-severe perio- } \\
\text { dontitis (30) } \\
\text { Mean (SD) }\end{array}$ & $62.96 \pm 17.24$ & $63.21 \pm 17.24$ & $5.3 \pm 0.5$ & $5.6 \pm 0.5$ \\
\hline$p$-Value ${ }^{d}$ & $<0.001^{\mathrm{a}, \mathrm{b}, \mathrm{c}}$ & $<0.001^{\mathrm{a}, \mathrm{b}, \mathrm{c}}$ & $<0.001^{c}$ & $<0.001^{c}$ \\
\hline
\end{tabular}

Abbreviation: SD, standard deviation.

apost hoc test was significant between healthy and mild P group.

bPost hoc test was significant between healthy and moderate to severe P group.

cPost hoc test was significant between mild and moderate to severe P group.

${ }^{\mathrm{d}} p<0.05$ is significant. 
Table 3 Mean values for salivary RANKL and OPG proteins in healthy, mild periodontitis and moderate to severe periodontitis groups

\begin{tabular}{|l|l|l|l|}
\hline Variable & $\begin{array}{l}\text { Salivary RANKL level }(\mathrm{ng} / \mathrm{mL}) \\
\text { Mean (SD) } n(67)\end{array}$ & $\begin{array}{l}\text { Salivary OPG } \\
\text { level }(\mathrm{ng} / \mathrm{mL}) \\
\text { Mean (SD) } \boldsymbol{n}(67)\end{array}$ & $p$-Value \\
\hline Healthy & $0.08(0.02)$ & $1.38(0.72)$ & $<0.001$ \\
\hline Mild periodontitis & $0.20(0.11)$ & $1.19(0.54)$ & $<0.001$ \\
\hline Moderate-to-severe periodontitis & $0.23(0.07)$ & $1.78(0.70)$ & $<0.001$ \\
\hline
\end{tabular}

Abbreviations: OPG, osteoprotegerin; RANKL, receptor activator of NF-KB ligand; SD, standard deviation.

Table 4 Association of clinical periodontal parameters with salivary RANKL and OPG protein levels in periodontitis patients

\begin{tabular}{|l|l|l|l|l|l|l|l|l|}
\hline & PPD & $p$-Value & CAL & $p$-Value & PS & $p$-Value & GBI & $p$-Value \\
\hline Salivary RANKL $(n=67)$ & $0.559^{\text {a }}$ & $<0.001$ & $0.554^{\text {a }}$ & $<0.001$ & $0.463^{\text {a }}$ & $<0.001$ & $0.537^{\text {a }}$ & $<0.001$ \\
\hline Salivary OPG $(n=67)$ & 0.112 & 0.367 & 0.077 & 0.534 & 0.121 & 0.330 & 0.091 & 0.466 \\
\hline
\end{tabular}

Abbreviations: CAL, clinical attachment level; GBI, gingival bleeding index; OPG, osteoprotegerin; PPD, periodontal pocket depth; PS, plaque score; RANKL, receptor activator of NF-KB ligand.

${ }^{a}$ Correlation is significant at the 0.01 level (two-tailed).

intergroup differences were observed to be nonsignificant for all the three study groups.

Pearson's coefficient correlation analysis was performed to assess the association between RANKL and OPG concentrations to periodontal parameters in patients with periodontitis. However, only RANKL salivary concentrations were noted for showing a significant, positive correlation with clinical periodontal parameters, and their variables in this study as depicted in - Table $\mathbf{5}$.

\section{Discussion}

Scientists have spent a long period of time attempting to establish a predictable molecular diagnostic marker of periodontal tissue destruction that can be utilized in clinical settings and possess high specificity and sensitivity. ${ }^{29}$ Several mediators that are responsible for alveolar bone remodeling experience constant washing into the saliva via GCF. Moreover, unstimulated whole saliva is seen as a dependable substitute to GCF sampling of isolated or specific sites due to the fact that it offers a more enhanced insight for the specific screening of analysis and proteins that are believed to induce the remodeling of bones within the periodontal microenvironment. ${ }^{30,31}$

Considering that an escalated salivary RANKL to OPG ratio plays a central part in the pathogenesis of periodontitis infection, ${ }^{32-34}$ a complete-mouth inspection was utilized to assess and evaluate if the salivary levels of RANKL and OPG proteins are correlated to the clinical outcomes of periodontitis. Thus, all study patients were sorted into three groups; those who do not have periodontitis disease or the group containing healthy patients (nonperiodontitis), patients with mild periodontitis group, and the moderate-to-severe periodontitis group. This study has shown that high RANKL and low OPG protein levels were found significant in patients suffering from severe periodontitis in comparison to nonperiodontitis patients (healthy group). Raised RANKL and OPG levels are responsible for net bone loss and degradation in advanced periodontal lesion. Also, the clinical periodontal parameters
(CAL, BOP, PS, and GBI) were more profound in severe periodontitis patients with high RANKL levels when compared with other groups of the study.

It appears that the RANKL and OPG protein levels observed for the saliva samples of both periodontal disease patients and those of healthy (nonperiodontitis) patients corresponded well with the levels detected in GCF samples. However, Frodge et al conducted a study that demonstrated that lower amounts of salivary RANKL was identified in majority of the salivary samples in their study. ${ }^{35}$ In part, one can attribute this discrepancy to the sensitivity of the immunoassay, variations in the sampling method, and sample size.

A statistically significant difference in terms of age, gender, race, PD, and CAL counts were observed in terms of clinical as well as demographic aspects of study participants in current study. Likewise, despite it become arduous to compare the outcomes of this study in contrast to other investigations owing to the variations in exploratory technique along with mathematical interpretation of the data.

The results of this study indicated that high RANKL levels were found in patients with moderate to severe periodontal disease in comparison with patients exhibiting mild periodontitis disease and healthy (nonperiodontitis) patients. In a similar manner, there were higher OPG proteins in the severe periodontitis group in contrast to the healthy (nonperiodontitis) group. The data of present study are consistent with all the previously available studies, which collectively indicate that RANKL is increased. However, OPG is decreased in periodontitis compared with healthy (nonperiodontitis) patients. ${ }^{36-38}$ However, no consensus or guideline exists in terms of the correlation between the RANKL and/or OPG concentrations/or values and the disease's clinical outcome in terms of CAL, PD, severity, and extent of periodontal degradation. Few earlier investigators including Mogi et al and Lu et $\mathrm{al}^{32,38}$ were unable to report significant correlations between GCF concentrations of RANKL and/or OPG and clinical measurements. Furthermore, the results of the current investigation agree with a previous report by Bostanci et al, which identified significant positive interrelationship for both 
Table 5 Multiple comparison of salivary RANKL and OPG proteins by using Dunnett's post hoc test

\begin{tabular}{|l|l|l|l|l|l|}
\hline & Groups & $\begin{array}{l}\text { Mean } \\
\text { difference }\end{array}$ & Standard error & $\begin{array}{l}\text { 95\% confidence } \\
\text { interval }\end{array}$ & $p$-Value \\
\hline Salivary & Healthy vs. mild & 0.12 & 0.25 & $0.05-0.18$ & $<-18$ \\
RANKL & Healthy vs. moderate to severe & 0.15 & 0.16 & $0.10-0.19$ & $<0.001$ \\
& Mild vs. moderate to severe & 0.03 & 0.02 & -0.42 to 0.10 & 0.6 \\
\hline Salivary & Healthy vs. mild & 0.18 & 0195 & -0.30 to 0.67 & 0.7 \\
OPG & Healthy vs. moderate to severe & 0.40 & 0.216 & -0.13 to 0.94 & 0.2 \\
& Mild vs. moderate to severe & 0.59 & 0.185 & $0.12-1.05$ & 0.009 \\
\hline
\end{tabular}

Abbreviations: OPG, osteoprotegerin; RANKL, receptor activator of NF-KB ligand. ${ }^{a} p<0.05$ is significant.

salivary RANKL and RANKL/OPG ratio and significant negative correlations for OPG in terms of periodontal clinical status. ${ }^{22}$

Despite the previously mentioned observations, both the smoking habit and the age of the patients satisfied the criteria for being classified as confounders, and therefore, it could have a significant influence on data interpretation and the results of the RANKL and OPG. The significant interrelationship between the clinical parameters and age, along with lower OPG values and higher RANKL values detected among the older patients, could also represent periodontal damage's aging-cumulative characteristics. These results coincide with previous studies, which exhibited significant age-dependent variations observed for RANKL and OPG levels, which potentially suggests that aging promotes the development of the osteoclast progenitor cells, significantly boosts the osteoblastic cell-influenced osteoclast formation and developmental process, changing the linkage and role between osteoclasts and osteoblasts in cancellous bone. ${ }^{39-41}$

For the prospective studies, we suggest that samples collected from different areas of Malaysia could provide a more generalized description of the prevalence of RANKL and OPG protein levels in periodontitis patients. Additionally, targeting patients diagnosed with only severe periodontitis disease would give a more detailed description of the influence of inflammatory pathway on the levels of salivary RANKL and OPG proteins. A longitudinal designed study would provide a better result for salivary RANKL and OPG in periodontitis patients, specially where postperiodontal therapy changes within the saliva could have been evaluated further as well.

Limitations observed for this study include:

- The study groups recruited patients from one particular state in Malaysia (Kelantan), whereas a diverse range of patients from different states might give a better representation of Malaysian population as a whole.

- The patients only consisted in majority of one type of race, that is, Malay and frequency of Chinese population was low. Representation from more Chinese and other races would give out a diverse representation in the results.

- Larger sample size would have provided a greater depth into the current hypothesis and results. The periodontitis patients in this study belonged to the mild and moderate to severe subcategories. Selecting only the patients with severe periodontitis might give a clearer picture of the level of inflammatory cytokines for diagnostic purposes.

- The diagnostic medium used was saliva, as opposed to plasma serum or GCF which, theoretically, are said to be having higher levels of inflammatory cytokines including RANKL and OPG.

\section{Conclusion}

The present study shows that salivary concentrations for RANKL, OPG proteins along with their ratio do not just benefit as an indicator of the extent and quantity of alveolar bone breakdown and PDL. Instead, there are numerous different confounding factors that could interact and influence the result of this disease, such as aging and different other habits of the susceptible host that may promote tissue destruction and the bone resorption in periodontitis as well.

\section{Note}

This study revealed that high RANKL levels are positively associated with periodontitis. High RANKL and low OPG levels can be potentially used as an aid in diagnosis of periodontitis clinical case. This work belongs to Department of Periodontics, School of Dental Sciences, Health campus Universiti Sains Malaysia, 16150 Kubang Kerian, Kelantan, Malaysia.

\section{Funding}

This study was supported by Research University Grant, Universiti Sains Malaysia (101/PPSG/8012353).

\section{Conflict of Interest}

None declared.

\section{Acknowledgments}

The authors are grateful to the School of Dental Sciences, Universti Sains Malaysia for permission and help in conducting this study. They would like to acknowledge my co-supervisors, staff of craniofacial laboratory, and staff of dental hospital for their technical support in sampling procedure and manuscript preparation.

\section{References}

1 Sanz M, van Winkelhoff AJ; Working Group 1 of Seventh European Workshop on Periodontology. Periodontal infections: understanding the complexity-consensus of the Seventh European Workshop on Periodontology. J Clin Periodontol 2011;38(Suppl 11):3-6

2 Socransky SS. Relationship of bacteria to the etiology of periodontal disease. J Dent Res 1970;49(2):203-222

3 Gurenlian JR. The role of dental plaque biofilm in oral health. J Dent Hyg 2007;81:116 
4 Hornef MW, Wick MJ, Rhen M, Normark S. Bacterial strategies for overcoming host innate and adaptive immune responses. Nat Immunol 2002;3(11):1033-1040

5 Graves DT, Cochran D. The contribution of interleukin-1 and tumor necrosis factor to periodontal tissue destruction. J Periodontol 2003;74(3):391-401

6 Hamza SA, Wahid A, Afzal N, et al. Effect of sodium bicarbonate mouth wash on salivary $\mathrm{pH}$ and interleukin- $1 \beta$ levels among smokers. Eur J Dent 2020;14(2):260-267

7 Boström L, Linder LE, Bergström J. Clinical expression of TNF- $\alpha$ in smoking-associated periodontal disease. J Clin Periodontol 1998;25(10):767-773

8 Kwan Tat S, Padrines M, Théoleyre S, Heymann D, Fortun Y. IL-6, RANKL, TNF-alpha/IL-1: interrelations in bone resorption pathophysiology. Cytokine Growth Factor Rev 2004;15(1):49-60

9 Takayanagi $\mathrm{H}$, Iizuka $\mathrm{H}$, Juji T, et al. Involvement of receptor activator of nuclear factor kappaB ligand/osteoclast differentiation factor in osteoclastogenesis from synoviocytes in rheumatoid arthritis. Arthritis Rheum 2000;43(2):259-269

10 Yasuda H, Shima N, Nakagawa N, et al. Osteoclast differentiation factor is a ligand for osteoprotegerin/osteoclastogenesis-inhibitory factor and is identical to TRANCE/RANKL. Proc Natl Acad Sci U S A 1998;95(7):3597-3602

11 Horwood NJ, Kartsogiannis V, Quinn JMW, Romas E, Martin TJ, Gillespie MT. Activated T lymphocytes support osteoclast formation in vitro. Biochem Biophys Res Commun 1999;265(1):144-150

12 Teng Y-TA, Nguyen H, Gao X, et al. Functional human T-cell immunity and osteoprotegerin ligand control alveolar bone destruction in periodontal infection. J Clin Invest 2000;106(6):R59-R67

13 Teitelbaum SL, Ross FP. Genetic regulation of osteoclast development and function. Nat Rev Genet 2003;4(8):638-649

14 Hsu H, Lacey DL, Dunstan CR, et al. Tumor necrosis factor receptor family member RANK mediates osteoclast differentiation and activation induced by osteoprotegerin ligand. Proc Natl Acad Sci U S A 1999;96(7):3540-3545

15 Yasuda H, Shima N, Nakagawa N, et al. Identity of osteoclastogenesis inhibitory factor (OCIF) and osteoprotegerin (OPG): a mechanism by which OPG/OCIF inhibits osteoclastogenesis in vitro. Endocrinology 1998;139(3):1329-1337

16 Kanzaki H, Chiba M, Shimizu Y, Mitani H. Periodontal ligament cells under mechanical stress induce osteoclastogenesis by receptor activator of nuclear factor kappaB ligand up-regulation via prostaglandin E2 synthesis. J Bone Miner Res 2002;17(2):210-220

17 Nakashima T, Kobayashi Y, Yamasaki S, et al. Protein expression and functional difference of membrane-bound and soluble receptor activator of NF-kappaB ligand: modulation of the expression by osteotropic factors and cytokines. Biochem Biophys Res Commun 2000;275(3):768-775

18 Theoleyre S, Wittrant Y, Tat SK, Fortun Y, Redini F, Heymann D. The molecular triad OPG/RANK/RANKL: involvement in the orchestration of pathophysiological bone remodeling. Cytokine Growth Factor Rev 2004;15(6):457-475

19 BoyceBF,XingL.FunctionsofRANKL/RANK/OPGinbonemodeling and remodeling. Arch Biochem Biophys 2008;473(2):139-146

20 Kong YY, Yoshida H, Sarosi I, et al. OPGL is a key regulator of osteoclastogenesis, lymphocyte development and lymph-node organogenesis. Nature 1999;397(6717):315-323

21 Mizuno A, Amizuka N, Irie K, et al. Severe osteoporosis in mice lacking osteoclastogenesis inhibitory factor/osteoprotegerin. Biochem Biophys Res Commun 1998;247(3):610-615

22 Bostanci N, Ilgenli T, Emingil G, et al. Gingival crevicular fluid levels of RANKL and OPG in periodontal diseases: implications of their relative ratio. J Clin Periodontol 2007;34(5):370-376
23 Vernal R, Dutzan N, Hernández M, et al. High expression levels of receptor activator of nuclear factor-kappa B ligand associated with human chronic periodontitis are mainly secreted by CD4+ T lymphocytes. J Periodontol 2006;77(10):1772-1780

24 Lu H-K, Chen Y-L, Chang H-C, Li C-L, Kuo MY-P. Identification of the osteoprotegerin/receptor activator of nuclear factor-kappa B ligand system in gingival crevicular fluid and tissue of patients with chronic periodontitis. J Periodontal Res 2006;41(4):354-360

25 Behfarnia P, Saied-Moallemi Z, Javanmard SH, Naseri R. Serum, saliva, and GCF concentration of RANKL and osteoprotegerin in smokers versus nonsmokers with chronic periodontitis. Adv Biomed Res 2016;5:80-80

26 Eke PI, Page RC, Wei L, Thornton-Evans G, Genco RJ. Update of the case definitions for population-based surveillance of periodontitis. J Periodontol 2012;83(12):1449-1454

27 Jasim H, Carlsson A, Hedenberg-Magnusson B, Ghafouri B, Ernberg M. Saliva as a medium to detect and measure biomarkers related to pain. Sci Rep 2018;8(1):3220

28 Al-Khtoum N, Qubilat AR, Al-Zaidaneen S. Al Mefleh S, Al-Qudah A. Clinical characteristics of pleomorphic adenoma of salivary glands among Jordanian patients. J Pak Med Assoc 2013;63(3):358-360

29 Buduneli N, Kinane DF. Host-derived diagnostic markers related to soft tissue destruction and bone degradation in periodontitis. J Clin Periodontol 2011;38(Suppl 11):85-105

30 Ng PY, Donley M, Hausmann E, Hutson AD, Rossomando EF, Scannapieco FA. Candidate salivary biomarkers associated with alveolar bone loss: cross-sectional and in vitro studies. FEMS Immunol Med Microbiol 2007;49(2):252-260

31 Sexton WM, Lin Y, Kryscio RJ. Dawson DR III, Ebersole JL, Miller CS. Salivary biomarkers of periodontal disease in response to treatment. J Clin Periodontol 2011;38(5):434-441

32 Mogi M, Otogoto J, Ota N, Togari A. Differential expression of RANKL and osteoprotegerin in gingival crevicular fluid of patients with periodontitis. J Dent Res 2004;83(2):166-169

33 Bostanci N, Saygan B, Emingil G, Atilla G, Belibasakis GN. Effect of periodontal treatment on receptor activator of NF-KB ligand and osteoprotegerin levels and relative ratio in gingival crevicular fluid. J Clin Periodontol 2011;38(5):428-433

34 Taubman MA, Kawai T, Han X. The new concept of periodontal disease pathogenesis requires new and novel therapeutic strategies. J Clin Periodontol 2007;34(5):367-369

35 Frodge BD, Ebersole JL, Kryscio RJ, Thomas MV, Miller CS. Bone remodeling biomarkers of periodontal disease in saliva. J Periodontol 2008;79(10):1913-1919

36 Sakellari D, Menti S, Konstantinidis A. Free soluble receptor activator of nuclear factor-kappab ligand in gingival crevicular fluid correlates with distinct pathogens in periodontitis patients. J Clin Periodontol 2008;35(11):938-943

37 Tang TH, Fitzsimmons TR, Bartold PM. Effect of smoking on concentrations of receptor activator of nuclear factor $\mathrm{\kappa B}$ ligand and osteoprotegerin in human gingival crevicular fluid. J Clin Periodontol 2009;36(9):713-718

38 Belibasakis GN, Bostanci N. The RANKL-OPG system in clinical periodontology. J Clin Periodontol 2012;39(3):239-248

39 Liu D, Xu JK, Figliomeni L, et al. Expression of RANKL and OPG mRNA in periodontal disease: possible involvement in bone destruction. Int J Mol Med 2003;11(1):17-21

40 Cao JJ, Wronski TJ, Iwaniec U, et al. Aging increases stromal/osteoblastic cell-induced osteoclastogenesis and alters the osteoclast precursor pool in the mouse. J Bone Miner Res 2005;20(9):1659-1668

41 Makhluf HA, Mueller SM, Mizuno S, Glowacki J. Age-related decline in osteoprotegerin expression by human bone marrow cells cultured in three-dimensional collagen sponges. Biochem Biophys Res Commun 2000;268(3):669-672 